\title{
Chromatin structure changes in Daphnia populations upon exposure to environmental cues - or - The discovery of Wolterecks "Matrix"
}

Ronaldo de Carvalho AUGUSTO

Ecole normale superieure de Lyon

Aki Minoda

Rikagaku Kenkyujo Yokohama Campus

Oliver Rey

Universite de Perpignan

Celine Cosseau

Universite de Perpignan

Cristian Chaparro

CNRS

Jeremie Vidal-Dupiol

Ifremer

Jean-Francois Allienne

CNRS

David Duval

Universite de Perpignan

Silvain Pinaud

Universite de Perpignan

Sina Tönges

Deutsches Krebsforschungszentrum

Ranja Andriantsoa

Deutsches Krebsforschungszentrum

Emilien Luquet

Universite de Lyon

Fabien Aubret

CNRS

Mamadou Dia Sow

Universite d'Orleans

Patrice David

CNRS

Vicki Thomson 
The University of Adelaide

Dominique Joly

CNRS

Mariana Gomes Lima

Fundacao Oswaldo Cruz

Deboraj Federico

CNRS

Etienne Danchin

CNRS

Christoph Grunau ( $\nabla$ christoph.grunau@univ-perp.fr)

Universite de Perpignan https://orcid.org/0000-0002-2161-412X

Research

Keywords: Epigenetics, ATAC-Seq, Daphnia pulex, norm of reaction, population epigenetics

Posted Date: May 29th, 2020

DOl: https://doi.org/10.21203/rs.3.rs-31260/v1

License: (c) (1) This work is licensed under a Creative Commons Attribution 4.0 International License. Read Full License 
Chromatin structure changes in Daphnia populations upon exposure to environmental cues - or - The discovery of Wolterecks "Matrix"

Ronaldo de Carvalho AUGUSTO ${ }^{\text {ab }}$, Aki MINODA ${ }^{\mathrm{c}}$, Oliver REY ${ }^{\mathrm{a}}$, Céline COSSEAU ${ }^{\mathrm{a}}$, Cristian CHAPARRO ${ }^{a}$, Jérémie VIDAL-DUPIOL ${ }^{a}$, Jean-François ALLIENNE ${ }^{a}$, David DUVAL $^{a}$, Silvain PINAUD ${ }^{a}$, Sina TÖNGES ${ }^{d}$, Ranja ANDRIANTSOA ${ }^{d}$, Emilien LUQUET $^{\mathrm{e}}$, Fabien AUBRET ${ }^{\mathrm{f}}$, Mamadou Dia SOW ${ }^{\mathrm{g}}$, Patrice DAVID ${ }^{\mathrm{h}}$, Vicki THOMSON', , Dominique JOLY ${ }^{j}$, Mariana GOMES LIMA ${ }^{k}$, Déborah FEDERICO ${ }^{1}$ Etienne DANCHIN ${ }^{1}$, Christoph GRUNAU ${ }^{\text {a1 }}$

${ }^{a}$ Univ. Perpignan Via Domitia, IHPE UMR 5244, CNRS, IFREMER, Univ. Montpellier, F66860 Perpignan, France.

${ }^{\mathrm{b}}$ Univ. Lyon, ENS de Lyon, Université Claude Bernard Lyon 1, Laboratoire de Biologie et Modélisation de la Cellule, CNRS, UMR 5239, INSERM, U1210, Lyon, 69007, France

${ }^{c}$ RIKEN, Epigenome Technology Exploration Unit, 1-7-22 Suehiro-cho, Tsurumi-ku, Yokohama, Kanagawa 230-0045, Japan.

d Division of Epigenetics, DKFZ-ZMBH Alliance, German Cancer Research Center, Im Neuenheimer Feld 580, 69120 Heidelberg, Germany

e Univ Lyon, Université Claude Bernard Lyon 1, CNRS, ENTPE, UMR5023 LEHNA, F69622, Villeurbanne, France

${ }^{\mathrm{f}}$ CNRS, Station d'Ecologie Théorique et Expérimentale, UMR 5321 CNRS - Université Paul Sabatier, 09200 Moulis, France/ School of Molecular and Life Sciences, Curtin University, Brand Drive, Bentley, WA 6102, Australia

g LBLGC, INRA, Université d'Orléans, EA1207 USC 1328, Orléans, France

${ }^{\mathrm{h}}$ CEFE, 1919 route de Mende, 34293 Montpellier, France

i School of Biological Sciences, University of Adelaide, Adelaide, South Australia 5005, Australia.

j Laboratoire Evolution, Génomes Comportement, Ecologie, CNRS Université Paris Sud UMR 9191, IRD UMR 247, Avenue de la Terrasse, 91198 Gif sur Yvette, France

k Laboratório de Malacologia, Instituto Oswaldo Cruz/Fiocruz, Pavilhão Adolpho Lutz, Av. Brasil 4365, 040-900 Rio de Janeiro, RJ, Brasil

${ }^{1}$ Laboratoire Évolution \& Diversité Biologique (EDB UMR 5174), Université Fédérale de Toulouse; CNRS, IRD. 118 route de Narbonne, Bât 4R1. 31062 Toulouse Cedex 9. France

${ }^{1}$ Correspondence: christoph.grunau@univ-perp.fr

\section{Abstract}

Background: This study therefore describes the classical experimental system postulated by Richard Woltereck 100 years ago: the adaptive morphological phenotypic plasticity of 
daphnia. Phenotypic plasticity is an important feature of biological systems that is likely to play a major role in the adaptation of organisms exposed to an environmental stimulus and it is increasingly related to epigenetic mechanisms. Several studies have started to identify the epigenetic basis of phenotypic plasticity of daphnia including non-coding RNAs, covalent modifications at the histone tails and DNA methylation however no study has yet investigated those effect on the genome-wide chromatin structure. The aim of this work was to study for the first time the overall genome-wide chromatin structure of Daphnia pulex in the context of the iconic complex defense response to predation. We developed a robust and rapid ATACseq technique that allows for analyzing chromatin of individual daphnia and show here (i) that this technique can be used with minimal expertise in molecular biology, and (ii) we used it to identify open chromatin structure in daphnia exposed to different environmental cues.

Results: Our results encouraged the expected induction of anti-predatory morphological changes in the stress treatment was significantly higher than that of daphnia from the control treatment. The developed ATAC-seq technique can be used to characterize chromatin structures of individuals even those that are small and thus with few biological material, making it possible to determine epigenetic polymorphisms relatively easily and at reasonable cost in full populations. In addition, we deliver evidence that chromatin structure changes upon stimuli from the environment.

Conclusion: We report here an extremely fast and straightforward method to map the chromatin status of individuals using small amounts of input biological material. We show here that changes in the environment, such as predator presence the chromatin structure is profoundly reorganised confirming Woltereck's classical postulate.

Keywords: Epigenetics, ATAC-Seq, Daphnia pulex, norm of reaction, population epigenetics 


\section{Background}

We are exploring here a classical battlefield of evolutionary biology. In a now landmark presentation in 1909, at the annual meeting of the German zoological society in Frankfurt, and at a time when oral presentations were still the major avenue for scientific exchange, "Mr. $R$. Woltereck (Leipzig)" exposed his ideas on Artveränderungen (change of species). The very same year, the scientific community had been captured by the publication of the German version of university lecture materials by the Danish botanist W. Johannsen, going back to 1903 [1]. Johannsen had introduced the terms of phenotype and genotype to separate the outer impressions we have of an organism from the heritable components it has inside. He also had introduced the notion of "pure lines" on which selection would be powerless since offspring of selected phenotypes would still produce the same range of phenotypes. His work was based on the mutation theory of de Vries [2] who had stated that the characters of organisms are made of distinct units that change spontaneously, salutatory, and relatively rarely. de Vries had called these changes Mutations. Mutations were in his eyes heritable and could be selected for. Woltereck's criticism was that the environment had no influence on de Vries "mutations" or the "exact science of heritability" of Johannsen. This was counterintuitive to Woltereck and many fellow scientists who saw that the environment had an impact on the phenotype. His battle horse became daphnia, easy to handle and cheap to maintain (contemporaries will understand the attractiveness of the system). Woltereck reasoned that, in response to Johannsen, further "analytical" experiments should also be done with "pure lines" (clonal lineages in modern terms), and with quantitative characters to investigate the role of the "milieu" (environment) on the character [3]. Woltereck recorded morphological measures, in particular the relative head length of his daphnia lines, depending on environmental conditions such as temperature and nutrition. These early studies paved the way to a subsequent rich literature that has documented the amazing property of daphnia to modify their phenotypes at the morphological, physiological, behavioral and more recently at the molecular levels in response to a large panel of environmental stressors including diet, pollution, heavy metals, and predator kairomones (reviewed in [4-5]). He called these relations of the phenotype on an environmental gradient Phänotypenkurve. The combined relations of a phenotypic character and all environmental gradients that influences it, he defined as Reaktionsnorm or "norm of reaction". According to him, norms of reaction are specific to species and to lineages within species, they are heritable and based on (in his opinion are equal to) the genotype. He postulated that reaction norms can progressively be shifted over generations depending on the culture 
conditions of daphnia [3]. Later, in his 1932 textbook (Woltereck was a lecturer at the University of Leipzig), he extended this view to the notion that species should be defined by identical norms of reaction [6]. This is a remarkable concept in the light of current difficulties to define species boundaries by phenotypic similarity, reproductive isolation or DNA sequence similarity. He also expanded the concept of the norm of reaction to three types of norms: 1st order (Modifikationen), 2nd order (Kombinationen), and 3rd order (Dauerinduktion and gene mutations). Modifikation was a textbook term in the 1920-30s and is equivalent to phenotypic variation. We will focus here on the 3rd order norms of reaction. Woltereck borrowed the term Dauerinduktion from Victor Jollos who had coined in the early 1900s; the term Dauermodifikation or "enduring modifications" [7], to describe phenotypic changes that could be provoked by environmental stimuli, would persist for a few generations and then revert.

Interestingly, Woltereck considered enduring modifications and gene mutations somehow similar. Nevertheless, he proposed that the molecular basis would be different. Mutations occurred in genes, while enduring modifications were based on something he called the Matrix. He suggested that this matrix was associated with the chromosomes ("... chromosomes are matrix plus gene..."), that it was heritable, changed during development of the organisms, and that changes of the matrix could be simple chemical substitutions of an unknown, but probably polymeric molecule. (More on Woltereck's work at https://embryo.asu.edu/pages/richardwolterecks-concept-reaktionsnorm and [8]). The phenomenon that organisms change their appearance as a function of environmental cues and/or during development is today rather called phenotypic plasticity, a term introduced in the 1960s. Mayr [9] used "polyphenism" to distinguish environmentally induced phenotypic variation from those that he believed were genetically determined (polymorphisms). Two years later, Bradshaw termed the amount by which the expression of an individual genotype can be modified by its environment as "plasticity" [10] and discussed the importance of plasticity for evolution. Nowadays, the importance of developmental and environmental plasticity for the generation of phenotypic novelty is still a matter of lively scientific discussion [11]. But it is increasingly recognized that enduring phenotypic plasticity requires memory effects that can be related to epigenetic mechanisms. The definition of what is epigenetic depends very much on the scientific context in which the term is used [12]. Here we will use it for any chromatin modification affecting gene expression, whether it is heritable or not [12] and we will show that it is related to Woltereck's matrix. 
In the last two decades, several studies have started to identify the molecular basis of such a matrix including non-coding RNAs, covalent modifications at the histone tails and DNA methylation. All of these mechanisms together constitute the epigenetic information that allows the remodeling (and maintenance) of chromatin structure and ultimately of phenotypes under environmental influence. In this regard, the global level of DNA methylation of Daphnia magna was found to be largely affected after exposure to abiotic (e.g. Zinc) and biotic (toxic cyanobacterium Microcystis aeruginosa, environmental toxicants, or to irradiation [13-15]. However, no study has yet investigated the effect of environmental stimuli on the genomewide chromatin structure. Here we argue that what Woltereck called the matrix is nowadays chromatin structure; the bearer of the overall epigenetic information including all epigenetic marks and their complex interactions. Here, we adapted an ATAC-Seq (Assay for Transposase Accessible Chromatin with high-throughput sequencing) [16]protocol to characterize the overall genome-wide chromatin structure of Daphnia pulex in the context of the iconic complex defense response to predation. ATAC-seq works similarly as DNase-seq (DNase I hypersensitive sites with high-throughput sequencing) [17], and determines which genomic regions are accessible to $\operatorname{Tn} 5$ transposase (i.e. open chromatin regions), especially the regulatory regions. Tn5 transposase inserts Illumina adapter sequences upon accessing the chromatin, which removes the need for additional steps to make the sequencing libraries later. This simple and efficient protocol reduces the enables starting material required, compared to DNase-seq. It also avoids many other steps such as the interaction with antibodies (e.g. ChIPseq) or chemical treatment (e.g. FAIRE-seq, WGBS) that might introduce bias.

Our results show: (i) that ATAC-seq can be used to characterize chromatin structures of individuals even those that are small and thus with few biological material, making it possible to determine epigenetic polymorphisms relatively easily and at reasonable cost in full populations; and (ii) we deliver evidence that chromatin structure changes upon stimuli from the environment (figure 1).

This study therefore describes the classical experimental system postulated by Richard Woltereck 100 years ago: the adaptive morphological phenotypic plasticity of daphnia. 


\section{$\underline{\text { Results }}$}

\section{ATAC-Seq can be used on individual Daphnia}

Our ATAC-seq procedure delivered reproducible chromatin profiles for individual daphnia. Projection of ATAC-seq reads on a metagene profile indicated that Tn5 accessible and thus presumably open chromatin structure occurs at the TSS and in gene bodies (Figure 2, suppl. Figure 1).

We started by comparing populations sampled at the beginning of the experiment (start) to the unexposed (control) population at the end of the exposure time of the experiment. Clustering algorithms built into DESeq2 were used to produce a graphical representation of sample-tosample distances based on the similarity of their ATAC-Seq patterns (Figure 3). These data indicate that there are 2,362 local differences (3.6\% of all 66,194 identified ATAC enrichment regions) at an FDR of 0.05 between the start and the control population (p-value adjusted for multiple testing with the Benjamini-Hochberg procedure).

This suggest that within 20 days (2-5 generations) there was either (i) epigenetic drift from 'start' to 'control' or (ii) epimutations were induced and/or selected by changes in the water tank environment from 'start' to 'control'.

\section{Exposure to predator cues leads to morphological differences in Daphnia}

Our results show that on average, the (LL-SL)/SL ratio calculated for daphnia from the stress treatment $(\mathrm{N}=14$; Mean $=0.24 \pm 0.072)$ was significantly higher than that of daphnia from the control treatment $(\mathrm{N}=12$; Mean $=0.15 \pm 0.039$; Mann-Whitney $\mathrm{U}$ Test, $U=19, \mathrm{Z}=-3.32$, $\mathrm{P}<0.001$ ) (Figure 4). This result confirms the expected induction of anti-predatory morphs in the stress treatment. It is noteworthy that the quantified morphological response to predation pressure observed in the stress treatment most likely reflects a more general response of stressed daphnia including morphological, physiological and behavioural changes [18]. Our first intention in comparing daphnia from the two experimental treatments was to confirm that we effectively induced a global response in stressed individuals, these responses having been otherwise much better documented previously [19]. 


\section{Exposure to predator cues leads to differences in chromatin structure between exposed}

(stressed) and unexposed (control) Daphnia

Using the DESeq2 procedure described above for 'start' vs. 'control' we identified 66,194 differences between 'control' and 'stressed'. This is by far too many, and indeed, shifts in MA plots (not shown) indicated that the assumption that is underlying the algorythm used in DESeq 2 and the requires that most sites do not change, was violated. Metagene profiles, using the same number of aligned reads over the entire genome, lend further support to the finding that 'stressed' samples had on average fewer reads over genes than 'control' samples indicating major changes in chromatin structure (Figure 5).

This also means that there is a large number of regions for which no reads could be recovered in the stressed samples. This is not due to a general lower accessibility of Tn5 to the cells and nuclei because of a thicker cuticle or a similar phenotypic trait because the insert size distribution of start, control and stressed populations are similar (Supplementary file 2). If DNA was more inaccessible in the stressed population we would expect longer fragments. To cope with the general decrease of ATAC-Seq reads in the stressed population, we resorted to ChromstaR, a HMM based software that was developed for ChIP-Seq analysis but that in principle can also be used for ATAC-Seq and is probably less sensitive to zero values. Under the constraints of numerous instances of an absence of data, ChromstaR identified 87 regions that are different between start and control, and stress. All were visually inspected using MACS2 average profiles, normalised by the same number of aligned reads over the genome. Among these 87 regions, ATAC signal was down in stressed samples compared to 'control and start' in 45 regions (52\%), down in 'stress and control' compared to 'start' in 16 (18\%), up in 'stress and control' in 3 (3.4\%), and down in 'control' in only 1 (1.1\%). Seven regions showed a heterogenous pattern on ATAC signals. In 15 regions differences were considered too weak (17\%) suggesting that fine tuning of ChromstaR parameters might be necessary (Supplementary file 2). These results are in line with a general decrease in ATAC signal in the stressed samples, i.e. chromatin becomes less accessible and/or less heterogenous. It is interesting to note that for 20 regions adjacent ATAC signals (less than $2 \mathrm{~kb}$ apart) were detected, lending further support to the idea that chromatin structure changes occur in a controlled fashion. Clustering of the samples clearly regroups control and stressed samples (Figure 6). 
Another way to cope with presence of many zeroes that could produce a difference between two groups simply because zeroes in one group turn out to be very small values in the other, is to use log transformation; setting an arbitrary low threshold level of accessibility that we do not consider very different from zero. Here we transformed the data with $\log 10(t+x)$, where $t$ is the threshold of 0.1 and $\mathrm{x}$ is the ATAC-seq read count. Doing so we see again that 'stressed' are very different from 'controls': the distribution has many very small values (including many true zeroes $=\log 10(\mathrm{t}+\mathrm{x})=-1)$ and 'stress' mode is slightly shifted to the left. Given that we used normalization, this must be compensated by a few sequences with very high numbers of reads (not visible but each counts a lot in the normalization). This suggests that under stress a few regions have many more reads than in controls and as a counterpart, many regions with relatively low number of reads have slightly less reads. By plotting transformed ATAC-Seq read counts of 'stressed' vs 'control' we see that there are two clouds of points: (i) those regions that have many more reads in stressed than in controls $(+1-2 \log 10$ units $=10-100$ fold change $)$ and (ii) under the 1:1 stressed-control line those regions that are slightly less represented in the 'stressed' than in the 'controls' (Figure 6). Regions identified by ChromstaR are present in both clouds (Figure 7).

We therefore conclude that stress modifies the distribution of the numbers of reads drastically: a few hundred regions are much more represented (i.e. Tn5 accessible) in stressed than in control chromatin. This could reflect the fact that under stress these regions become accessible in many more tissues than in control conditions, thus they are captured many more times by the ATAC-seq. As a counterpart, the proportional representation of most other regions slightly decreases (fold change approximately $1 / 2=-0.3 \log 10$ units) but remain generally proportional to their value in controls. Competition for sequencing (PCR amplification selects for the many reads from highly accessible regions) and normalization (divide by the total number of sequences) can be responsible for this.

\section{$\underline{\text { Discussion }}$}

We report here a very fast and straightforward method to map the chromatin status of individuals using small amounts of input biological material [20]. The technique is very robust and we have been using it for more than a year now on different species e.g. adult worms of the parasite Schistosoma mansoni. The technique was successfully used in the framework of a summer school for field ecologists, some of them with no training in molecular biology. The technique avoids many caveats that are involved with the use of antibody-based methods [21] 
and is roughly 6 times faster. In our hands, there was no problem with mitochondrial contamination which is sometimes observed with other ATAC-seq methods. However, it also has some drawbacks: when using aquatic organisms, we observed DNA pollution from other species than the model species/species of interest. It is thus necessary to carefully wash the samples in DNA-free water. In addition, organisms should not be fed a couple of days before the ATAC experiment is performed. Another, inherent weakness of the method is that it provides just a positive readout of Tn5 accessible. These regions and considered nuleosomefree and presumably euchromatic. Absence of ATAC-Seq reads is generally considered as signal for inaccessible, and therefore heterochromatic regions. However, as with any method, the absence of proof is not proof of absence. This caveat is of course shared with any technique that relies on enzymatic accessibility such as DNA footprint, DNAse-seq or FAIRE-seq. It would be desirable to develop techniques that also provide a positive display of heterochromatic regions and without any use of antibodies. Notwithstanding these caveats, due to its minimal training requirements, low starting material as input and price advantage over other techniques, ATAC-seq can be used to develop fast epigenotyping approaches in populations similar to what is done routinely today in population genetics.

We estimate here the epimutation rate to be at least in the order of magnitude of $10^{-3}$ which is in line to earlier findings [22-23]. However, we also realize that the current analysis methods are not suitable if strong, global changes in chromatin structure occurs. As for genetic analyses, the underlying assumptions of algorithms is that (epi)mutations are rare events. This prompts a need to develop new analysis methods that take large genome wide modifications of the chromatin structure into account. HMM based methods or our transformation method are promising starting points for this purpose.

The development of defensive crests upon exposure to kairomones from predators has been studied in great detail in several Daphnia species. It's developmental dynamics [24] and even the genes that are differently expressed [25] are now known. It is also recognized that genetic, non-genetic and environmental elements interact to bring about phenotypic variation in daphnia populations [26], a phenomenon that is probably applicable to all eukaryotes and that we have recently conceptualized as a systems biology view on inheritance [27]. Daphnia possess bearers of epigenetic information such as modified histones [28], and DNA methylation [29] of the classical mosaic type [30]. Despite the the fact that the idea of a matrix was known when the term 'phenotypic plasticity' was formalised, it remains surprising that, to 
date, the link between Woltereck's matrix and the chromatin has not been explicitly made. Maybe because it was too evident, or maybe because the idea that heritable units are composed of several elements and not only DNA fragments (in Woltereck's words “... chromosomes are matrix plus gene...") is still not entirely accepted by the scientific community. The matrix concept provides a clear key to understanding how organisms can interpret environmental cues and change their phenotype over time spans that are beyond the duration of the cue. In terms of systems biology, the capacity to interpret these cues is the 'emerging property' of the inheritance system that is composed of genotype and epigenotype (and potentially cytoplasmic elements and microorganisms). It also allowed eukaryotic cells to generate complexity and thus the symbiontic acquisition of histone-based chromatin organization was probably critical for the evolution of eukaryotic complex cells [31].

\section{Conclusion}

In conclusion, we show here that moderate changes in the environment (during the 20 days, i.e. 2-5 generations, from 'start' where daphnia where introduced into their new water tanks to 'control' ) are accompanied by roughly $4 \%$ of epigenetic modifications. When strong environmental cues, such as predator presence, are applied, the chromatin structure is reorganised much more profoundly and many regions become inaccessible to Tn5.

\section{Methods}

\section{Daphnia culture and experimental design}

A batch of $\sim 300$ commercial Daphnia pulex was obtained from a commercial supplier (Aqualiment: http://www.aqualiment.eu/). At their arrival, daphnia were immediately split into two sets of equal size $(\sim 150 \times 2)$ and placed in two independent experimental tanks (i.e. initial density of 75 ind. $L^{-1}$ ), hereafter called the 'stress' and the 'control' tanks. Each experimental tank consisted in a2-L plastic aquaria ( $\mathrm{L} \times 1 \times \mathrm{h}=18 \times 12 \times 11 \mathrm{~cm})$ supplied with clean water, inside of which a floating plastic fish breeding isolation box $(\mathrm{L} \times 1 \times \mathrm{h}=12,5 \times 8 \times 7 \mathrm{~cm})$ was placed (Figure 1). These isolation boxes are transparent with a series of $1 \mathrm{~mm}$ cracks on the bottom wall to allow water connection between the tanks and inside the isolation box. Daphnia were acclimated in their respective experimental tanks out of the isolation box for 20 days prior to starting the experiment. This lag time before the experiment also allowed the production of new daphnia offspring born in our experimental setup. During this acclimating period, only negligible mortality was observed and newly hatched daphnia were observed in the two 
experimental tanks. After this 20-day acclimating period, a predator (i.e. a guppy fish previously trained to eat daphnia) was introduced into the isolation box of one experimental tank during 15 days (i.e. hereafter called the 'stress treatment', compared to the 'control treatment'). During the experiment the fish was fed every other day with 10 daphnia collected alternatively from the stress and the control tank (i) to avoid subsequent biases in density between the experimental treatments and (ii) to account for a possible effect of daphnia sampling on congeners' responses. Daphnia sampling for fish feeding was achieved using a sterile 3-ml plastic transfer pipet. This experimental setup allowed the daphnia of the stress treatment to experience an indirect predation pressure (i.e. without predation risk) through a direct visual contact with the predator and an olfactory contact with environmental cues released by the predator. Overall the experiment, the daphnia and the predator were maintained at room temperature following the natural photoperiod and the former were fed ad libitum with clean phytoplancton (i.e. chlorellasp.) reared in our lab facilities.

\section{Sampling and morphometry}

Four daphnia were sampled during the 20 days acclimating period (called herein 'start' population) and immediately processed for ATAC-Seq. At day 15 of stress treatment (2-5 generations), 12 and 14 living daphnia were respectively sampled from each of the control and stress treatment by pipetting through a $1 \mathrm{~mL}$ automatic pipette with enlarged openings of the pipetting tips and disposed on microscopic slides for dark field microscopy. To avoid experimenter bias 10 different persons sampled at least one control and one stressed daphnia. Each daphnia was observed and photographed under a stereo microscope (Leica EZ4) at a 100 fold magnification using the Leica application suite LAS EZ Version 3.4.0.

From each picture two body lengths were measured (Figure 8): the short length $(\mathrm{SL}=$ from the middle of the eye to the base of the apical spine) and the long length ( $L L=$ from the middle of the eye to the tip of the apical spine). Finally, each measured animal was then individually transferred to a $1.5 \mathrm{~mL}$ Eppendorf tube and was immediately processed for ATAC-seq library preparation. To check for morphological response of daphnia to predation pressure we compared the individual ratio of (LL-SL)/SL of each treatment with a student t-test using Excel and http://www.estimationstats.com/\#/analyze/two-independent-groups. 
The ATAC-Seq protocol we used is based on [32] with some modifications [20]. All water was removed from the daphnia containing tubes through pipetting. Daphnia were washed once with $50 \mu 1$ cold PBS and all liquid was then removed by pipetting. $22 \mu 1$ nuclease free water, $25 \mu 1$ 2x TD buffer (Illumina FC-121-1030), $2.5 \mu$ TDE (Tn5 Illumina FC-121-1030) and $0.5 \mu 1$ 1\% IGEPAL CA-630 (Sigma-Aldrich, cat. no. I8896) were added and mixed by pipetting 10 times to disrupt cells. Reaction mixtures were incubated at $37^{\circ} \mathrm{C}$ for $30 \mathrm{~min}$ at $300 \mathrm{rpm}$ agitation. Transposed DNA was immediately purified using a QIAquick PCR Purification Kit (\#28106), and purified DNA was eluted into $10 \mu \mathrm{l}$ of elution buffer (10 mMTris-HCl, $\mathrm{pH} 8)$. Libraries were PCR amplified using Promega GoTaq2, universal Ad1_noMX primer and index primer Ad2.* (http://www.nature.com/nmeth/journal/v13/n11/extref/nmeth.3999-S5.xlsx) (each 1.25 $\mu \mathrm{M})$ that was different for each individual daphnia, $10 \mu \mathrm{l}$ of DNA in a total volume of $50 \mu \mathrm{l}$. Pre-amplification was done at $98^{\circ} \mathrm{C}$ for $30 \mathrm{sec}$, then five cycles of $98^{\circ} \mathrm{C}$ for $10 \mathrm{sec}, 63^{\circ} \mathrm{C}$ for $30 \mathrm{sec}, 72^{\circ} \mathrm{C}$ for $1 \mathrm{~min} .5 \mu 1$ of this PCR mixture was used for qPCR analysis to determine the number of additional amplification cycles. Relative fluorescence was plotted versus cycle number and the cycle number that corresponds to one-third of the maximum fluorescent intensity was used for additional PCR amplification. After PCR, size-selection at $300 \mathrm{bp}$ was done on an IP-Star system with Ampure XP beads. Quality and quantity of libraries were checked with an Agilent Bioanalyzer High Sensitivity DNA Assay and library were sequenced on a NextSeq550 High Output Flowcell as paired-end and 75 bp. A detailed step-by-step protocol in Augusto et al 2019.

\section{Detection of chromatin structure differences}

Sequence quality was checked with FastQC (http://www.bioinformatics.babraham.ac.uk/projects/fastqc/). Reference genome was downloaded from $\quad \underline{\mathrm{ftp}}$ //ftp.ensemblgenomes.org/pub/metazoa/release40/fasta/daphnia_pulex/dna/Daphnia_pulex.V1.0.dna.toplevel.fa.gz _, corresponding to GenBank assembly accession GCA_000187875.1. Alignment was done with Bowtie2 evoking the following parameters: bowtie2-align-s basic-0 -p 6 -x genome -N 1 -L 32 -i S,1,1.15 --nceil L,0,0.15 --dpad 15 --gbar 4 --end-to-end --score-min L,-0.6,-0.6. Uniquely aligned reads were retained by filtering the tag "XS:i:" that is absent in their alignement annotations. 
For visualisation of ATAC profiles all BAM files for each condition were merged, converted to header-free SAM, and downsampled with a custom script that draws random lines to 409,000 aligned reads. This corresponds to the condition with the lowest number of aligned reads. For analysis of individual daphnia PCR duplicates were removed with SamTools RmDup. Bedgraph files were generated with MACS2 using model building, lower fold bound of 5, upper fold bound 50, band width $300 \mathrm{bp}$, minimum FDR for peak detection of 0.05 , an effective genome size of $150,000,000$, and without calling broad regions. Bedgraphs were loaded into IGV for visual inspection. For analysis of individual daphnia, background correction was done with MACS bdgcmp. Bedgraph was converted into BigWig. The DeepTools suite was used for representation of metagene profiles based on this over 15,287 genes on the forward strand. Gene annotation files were downloaded from ftp://ftp.ensemblgenomes.org/pub/metazoa/release-

40/fasta/daphnia_pulex/cds/Daphnia_pulex.V1.0.cds.all.fa.gz . More information is available at https://metazoa.ensembl.org/Daphnia_pulex/Info/Annotation/

Two different approaches were used for further data analysis. One uses a combination of peakcalling with MACS2, extraction of read coverage in peaks with BEDtools, and DESeq2 for differential analysis. To detect all peak regions for all conditions, BAM files of control and stress conditions were merged and peakcalling was performed with MACS2 as described above. The number of reads overlapping peak regions was extracted with bedtools intersect -a peakfile.bed -b individual_bam_files.bam -header -wa -c, Columns 4 and 11, corresponding to peak-names and number of overlapping features, i.e. coverage were used as input for DESeq2. All analyses were done at the galaxy instance of the Labex CeMEB/IHPE (http://bioinfo.univperp.fr).

The second approach was based on Hidden-Markow-Models (HMM) implemented in ChromstaR (v.1.2.0) for genome-wide characterization of open chromatin landscape. On this approach control and stress condition were processed in two steps: (1) we fitted a univariate HMM over each ATAC-seq samples individually and (2) we performed a multivariate HMM over the combined ATAC-seq samples in each condition. For that, BAM files were processed under the differential mode, with a false discovery rate (FDR) cutoff of 0.05 and bin size of 500. 


\section{End Matter}

\section{Ethics approval and consent to participate}

No ethics approval was necessary.

\section{Author Contributions and Notes}

R.A. and C.G. designed research, all authors performed research, P.D. wrote software, R.A., P.D. and C.G. analyzed data; and all authors wrote the paper.

\section{Competing interests}

The authors declare no conflict of interest.

\section{Availability of data and materials}

This article contains supporting information online on NCBI SRA (BioProject PRJNA587385). Correspondence between sample names used in this text and accession numbers are in table 1.

\section{Consent for publication}

Not applicable

\section{Acknowledgments and Funding}

These experiments were done in the framework of the RTP 3E summer school "Epigenetics for field ecologists" and received support from the CNRS.

\section{References}

1. Johannsen W (1909) Elemente der Exakten Erblichkeitslehre.

2. Vries HD, MacDougal DT (1905) Species and varieties, their origin by mutation; lectures delivered at the University of California.

3. Woltereck R (1909) Weitere experimentelle Unter-suchungen uber Artveranderung, speziell uber das Wesen quantitativer Artunterschiede bei Daphniden. Verh. D. Tsch. Zool. Ges., 1909, 110-172.

4. Riessen H (2011) Predator-induced life history shifts in Daphnia: A synthesis of studies using meta-analysis. Canadian Journal of Fisheries and Aquatic Sciences, 56, 24872494. 
5. Harris KD, Bartlett NJ, Lloyd VK (2012) Daphnia as an emerging epigenetic model organism. Genet Res Int, 2012, 147892.

6. Woltereck R (1932) Grundzüge Einer Allgemeinen Biologie. Die Organismen Als Gefüge, Getriebe, Als Normen und Als Erlebende Subjekte.

7. Jollos V (1939) Grundbegriffe der Vererbungslehre, insbesondere Mutation, Dauermodifikation, Modifikation.

8. Nicoglou A (2017) The concept of plasticity in the history of the nature-nurture debate in the early twentieth century. pp. 97-122.

9. Mayr E (1963) Animal species and evolution. Animal species and evolution.,

10. Bradshaw AD (1965) Evolutionary Significance of Phenotypic Plasticity in Plants. In: 13, (eds Caspari EW, Thoday JM), pp. 115-155. Academic Press,

11. Levis NA, Pfennig DW (2019) Phenotypic plasticity, canalization, and the origins of novelty: Evidence and mechanisms from amphibians. Semin. Cell Dev. Biol., 88, 8090 .

12. Nicoglou A, Merlin F (2017) Epigenetics: A way to bridge the gap between biological fields. Studies in History and Philosophy of Science Part C

13. Vandegehuchte MB, Vandenbrouck T, De Coninck D, De Coen WM, Janssen CR (2010) Gene transcription and higher-level effects of multigenerational $\mathrm{Zn}$ exposure in Daphnia magna. Chemosphere, 80, 1014-1020.

14. Asselman J, De Coninck DI, Beert E et al. (2017) Bisulfite Sequencing with Daphnia Highlights a Role for Epigenetics in Regulating Stress Response to Microcystis through Preferential Differential Methylation of Serine and Threonine Amino Acids. Environ. Sci. Technol., 51, 924-931.

15. Trijau M, Asselman J, Armant O, Adam-Guillermin C, De Schamphelaere KAC, Alonzo F (2018) Transgenerational DNA Methylation Changes in Daphnia magna Exposed to Chronic $\gamma$ Irradiation. Environ. Sci. Technol., 52, 4331-4339.

16. Buenrostro JD, Giresi PG, Zaba LC, Chang HY, Greenleaf WJ (2013) Transposition of native chromatin for fast and sensitive epigenomic profiling of open chromatin, DNAbinding proteins and nucleosome position. Nature Methods, 10, 1213 EP -.

17. Song L, Crawford GE (2010) DNase-seq: a high-resolution technique for mapping active gene regulatory elements across the genome from mammalian cells. Cold Spring Harb Protoc, 2010, pdb.prot5384.

18. Boersma M, De Meester L, Spaak P (1999) Environmental stress and local adaptation in Daphnia magna. Limnology and Oceanography, 44, 393-402.

19. Riessen HP (1999) Predator-induced life history shifts in Daphnia: a synthesis of studies using meta-analysis. Canadian Journal of Fisheries and Aquatic Sciences, 56, 2487-2494. 
20. Augusto R, Minoda A, Grunau C (2019) A simple ATAC-seq protocol for population epigenetics, BioRxiv

21. Egelhofer TA, Minoda A, Klugman S et al. (2011) An assessment of histonemodification antibody quality. Nat Struct Mol Biol, 18, 91-93.

22. van der Graaf A, Wardenaar R, Neumann DA et al. (2015) Rate, spectrum, and evolutionary dynamics of spontaneous epimutations. Proc Natl Acad Sci U S A, 112, 6676-6681.

23. Roquis D, Rognon A, Chapparo C et al. (2016) Frequency and mitotic heritability of epimutations in Schistosoma mansoni. Mol Ecol, 25, 1741-1758.

24. Weiss LC, Leimann J, Tollrian R (2015) Predator-induced defences in Daphnia longicephala: location of kairomone receptors and timeline of sensitive phases to trait formation. J. Exp. Biol., 218, 2918-2926.

25. Rozenberg A, Parida M, Leese F, Weiss LC, Tollrian R, Manak JR (2015) Transcriptional profiling of predator-induced phenotypic plasticity in Daphnia pulex. Frontiers in zoology, 12, 18.

26. Harney E, Paterson S, Plaistow SJ (2017) Offspring development and life-history variation in a water flea depends upon clone-specific integration of genetic, non-genetic and environmental cues. Functional Ecology, 31, 1996-2007.

27. Cosseau C, Wolkenhauer O, Padalino G, Geyer KK, Hoffmann KF, Grunau C (2016) (Epi)genetic Inheritance in Schistosoma mansoni: A Systems Approach. Trends Parasitol, 33, 285-294.

28. Robichaud NF, Sassine J, Beaton MJ, Lloyd VK (2012) The Epigenetic Repertoire of Daphnia magna Includes Modified Histones. Genet Res Int, 2012, 174860.

29. Kvist J, Gonalves Athansio C, Shams Solari O et al. (2018) Pattern of DNA Methylation in Daphnia: Evolutionary Perspective. Genome Biol Evol Genome biology and evolution, 10, 1988-2007.

30. Aliaga B, Bulla I, Mouahid G, Duval D, Grunau C (2019) Universality of the DNA methylation codes in Eucaryotes. Scientific Reports, 9, 173.

31. Brunk CF, Martin WF (2019) Archaeal Histone Contributions to the Origin of Eukaryotes. Trends Microbiol., 27, 703-714.

32. Corces MR, Buenrostro JD, Wu B et al. (2016) Lineage-specific and single-cell chromatin accessibility charts human hematopoiesis and leukemia evolution. Nat Genet, 48, 1193-1203. 


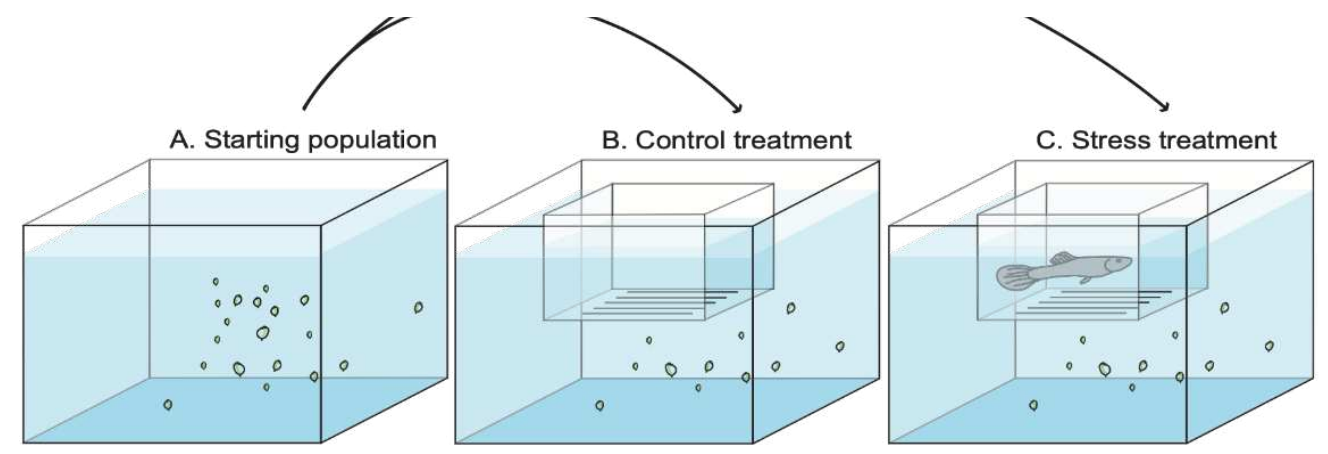

Figure 1: Experimental design used in this study. Daphnia were put into a water tank and allowed to acclimate (start population). Then, two experimental tanks were set up following strictly the same design. The only difference was the presence of a predator (a guppy trained to eat daphnia) in the floating plastic fish breeding isolation box in the stress treatment. 


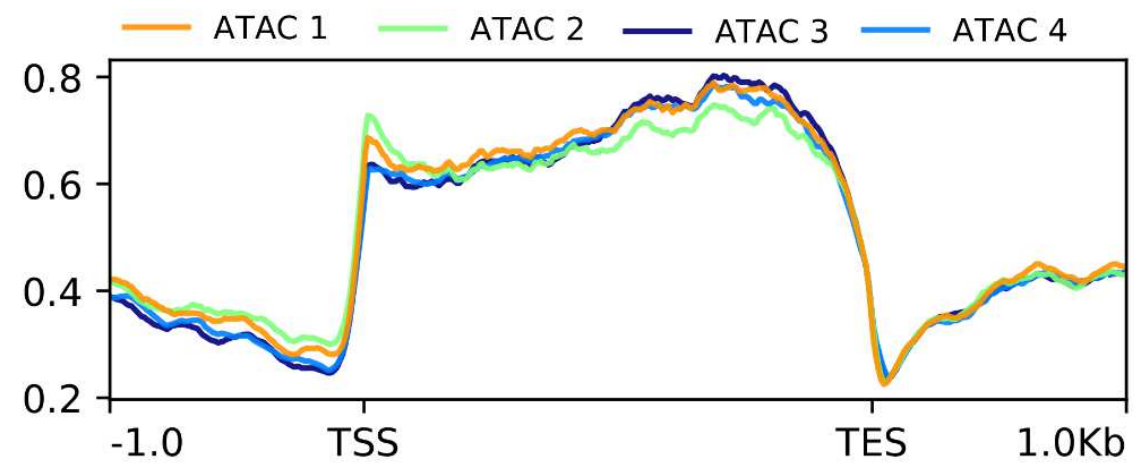

Figure 2: Superposed metagene ATAC profiles of four individual daphnia of the start populations. X-axis in base-pairs. TSS = Transcription start site, TES = transcription end site. Y-axis average enrichment of ATACseq reads over genes and upstream and downstream regions. Enrichment of accessible chromatin occurs along the entire length of the genes. 


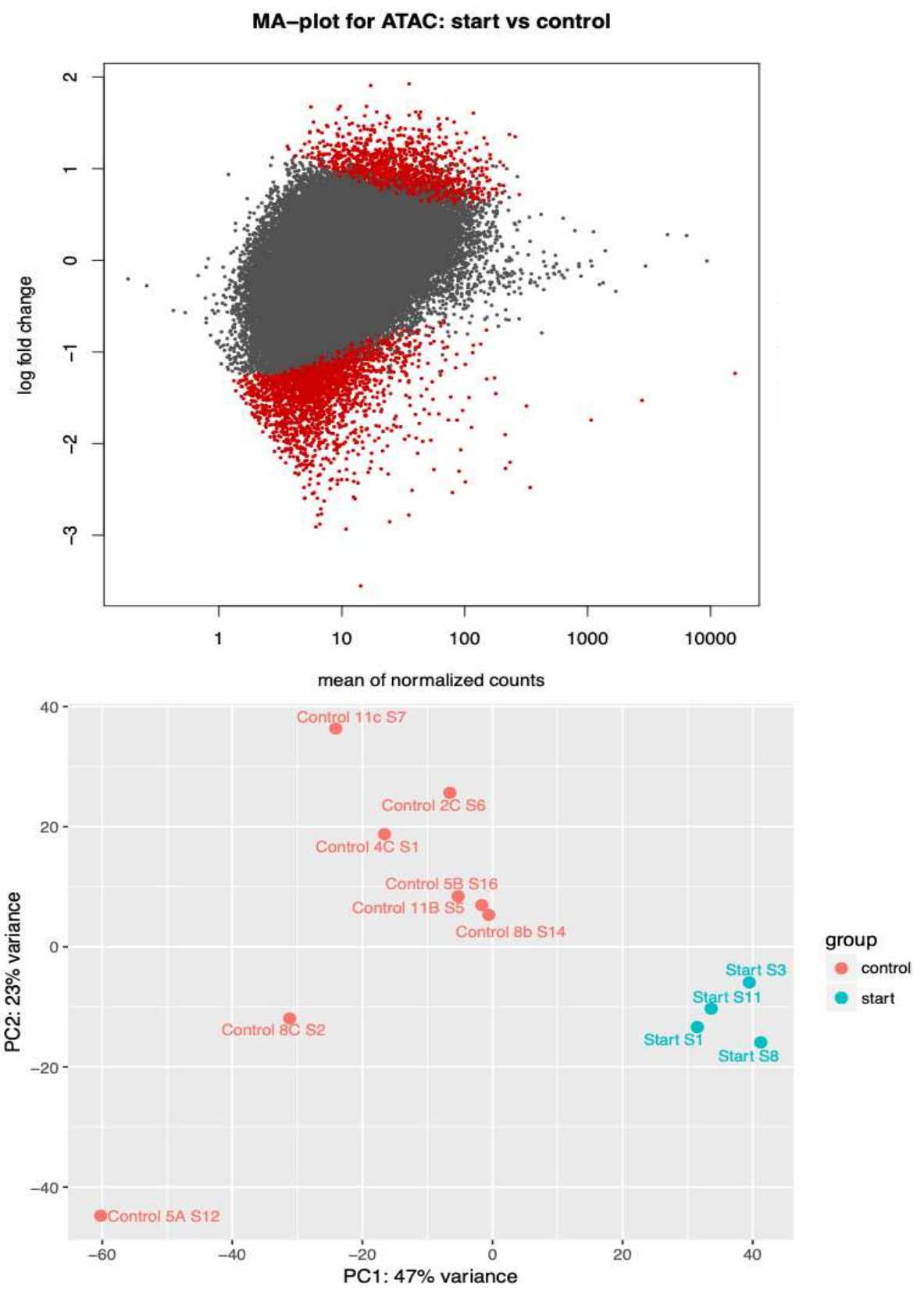

Figure 3: MA-Plot (top) and Principal component analysis of individual Daphnia based on their ATACSeq profiles (bottom). On the PCA every point represents an individual daphnia. Populations are color coded. Samples from the start (blue) and control population (red) cluster clearly. 


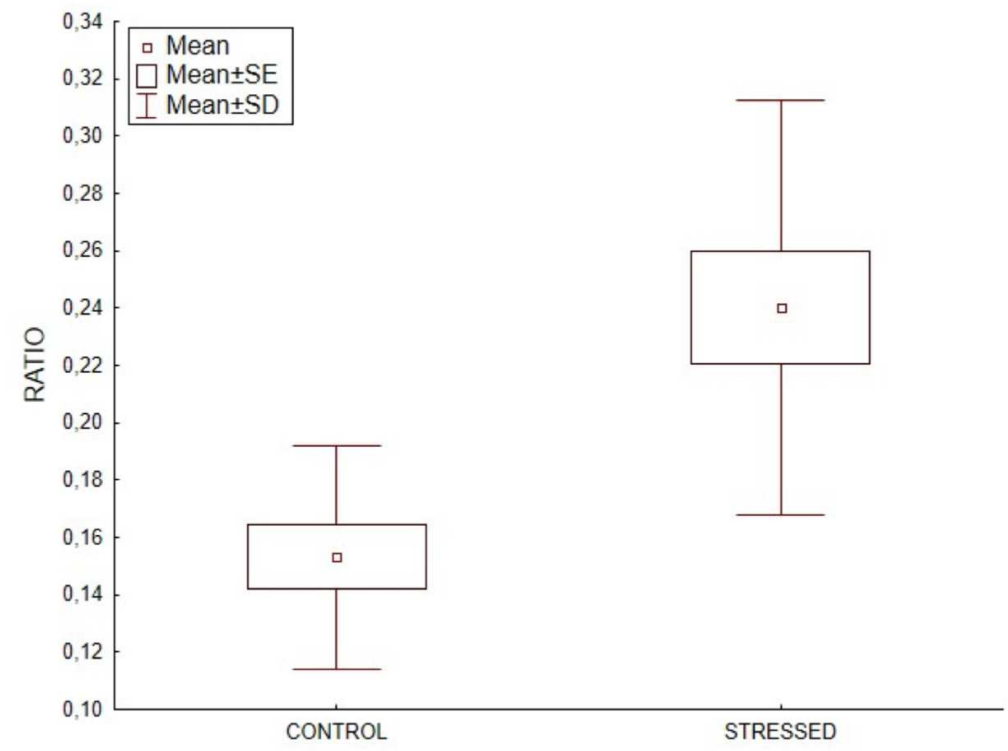

Figure 4: Boxplot of morphometric ratios of (LL-SL)/SL in control and stressed daphnia populations (control: $\mathrm{N}=12$; stress: $\mathrm{N}=14$ ). 


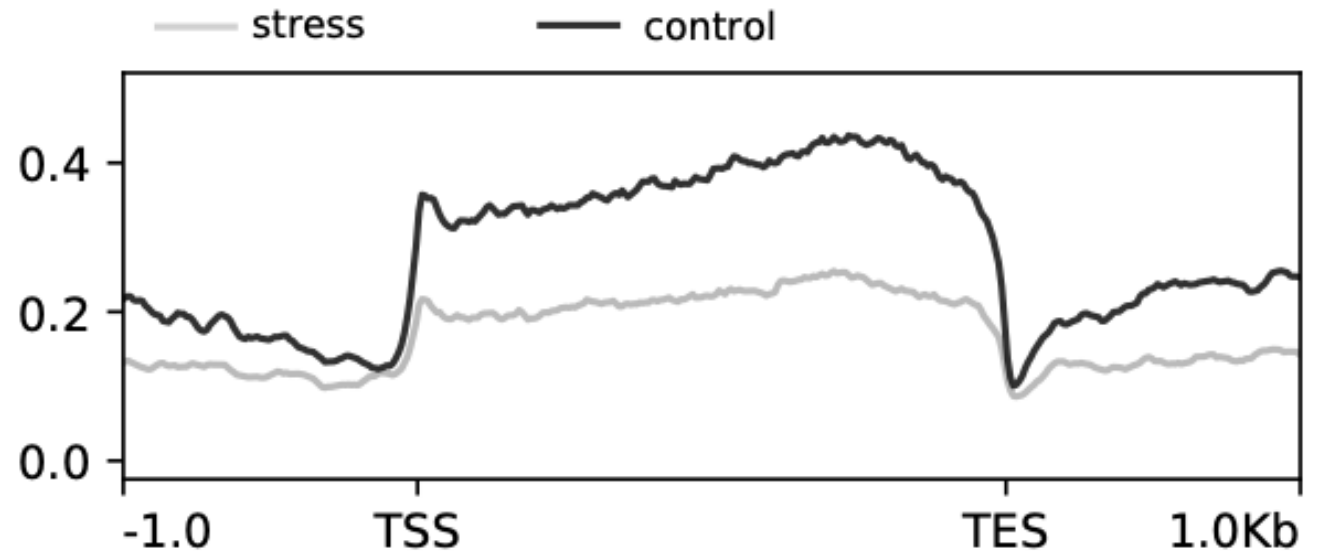

Figure 5: Combined metagene ATAC profiles of stressed and control daphnia populations. $\mathrm{X}$-axis in basepairs. TSS $=$ Transcription start site, TES $=$ transcription end site. $Y$-axis average enrichment of ATAC-seq reads over genes and upstream and downstream regions. 


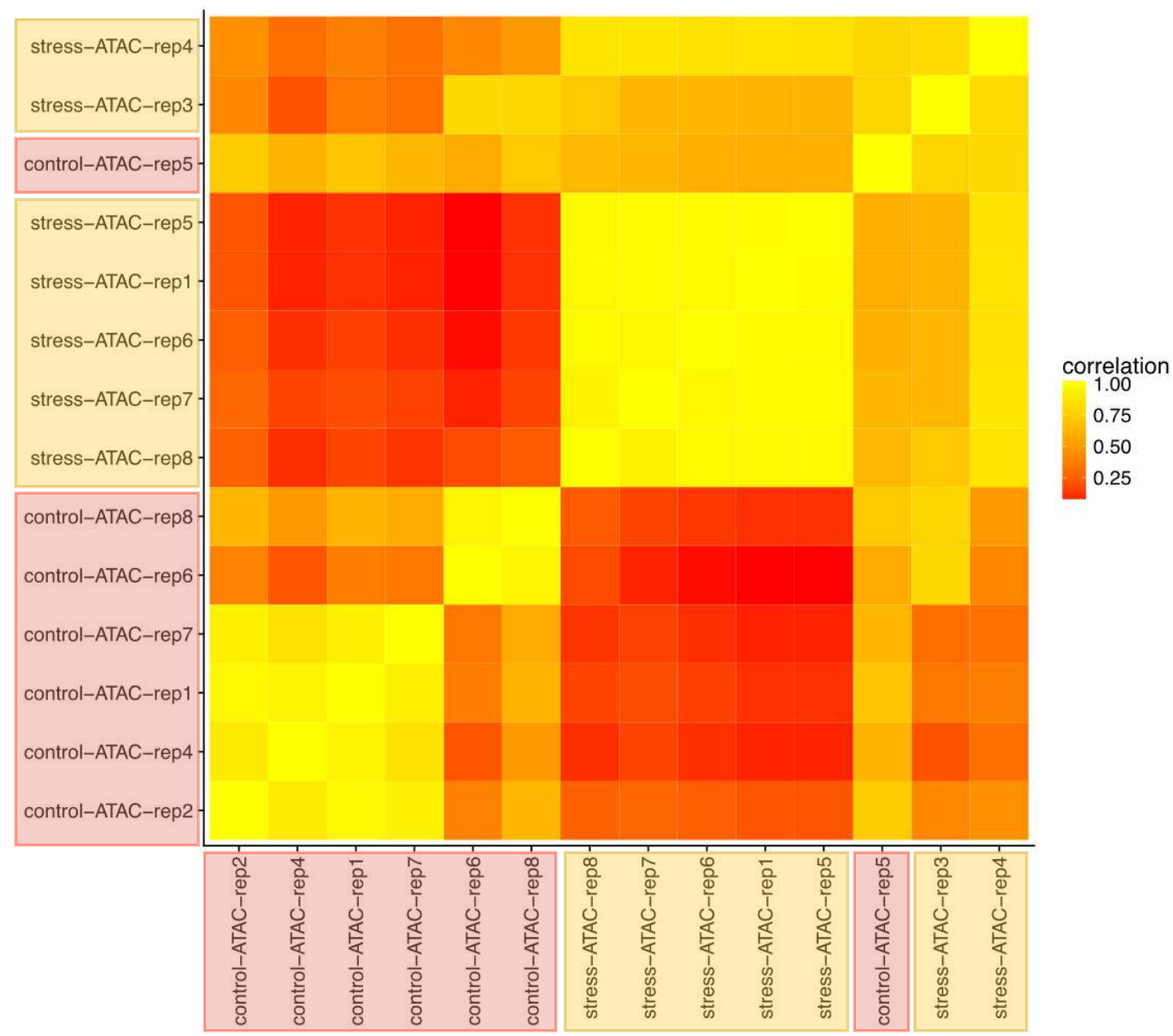

Figure 6: Clustering of individual daphnia based on their ATAC-Seq profiles. Heat map indicating similarity in the HMM ChromstaR results. Generally samples from the stressed and the control populations each cluster together. 


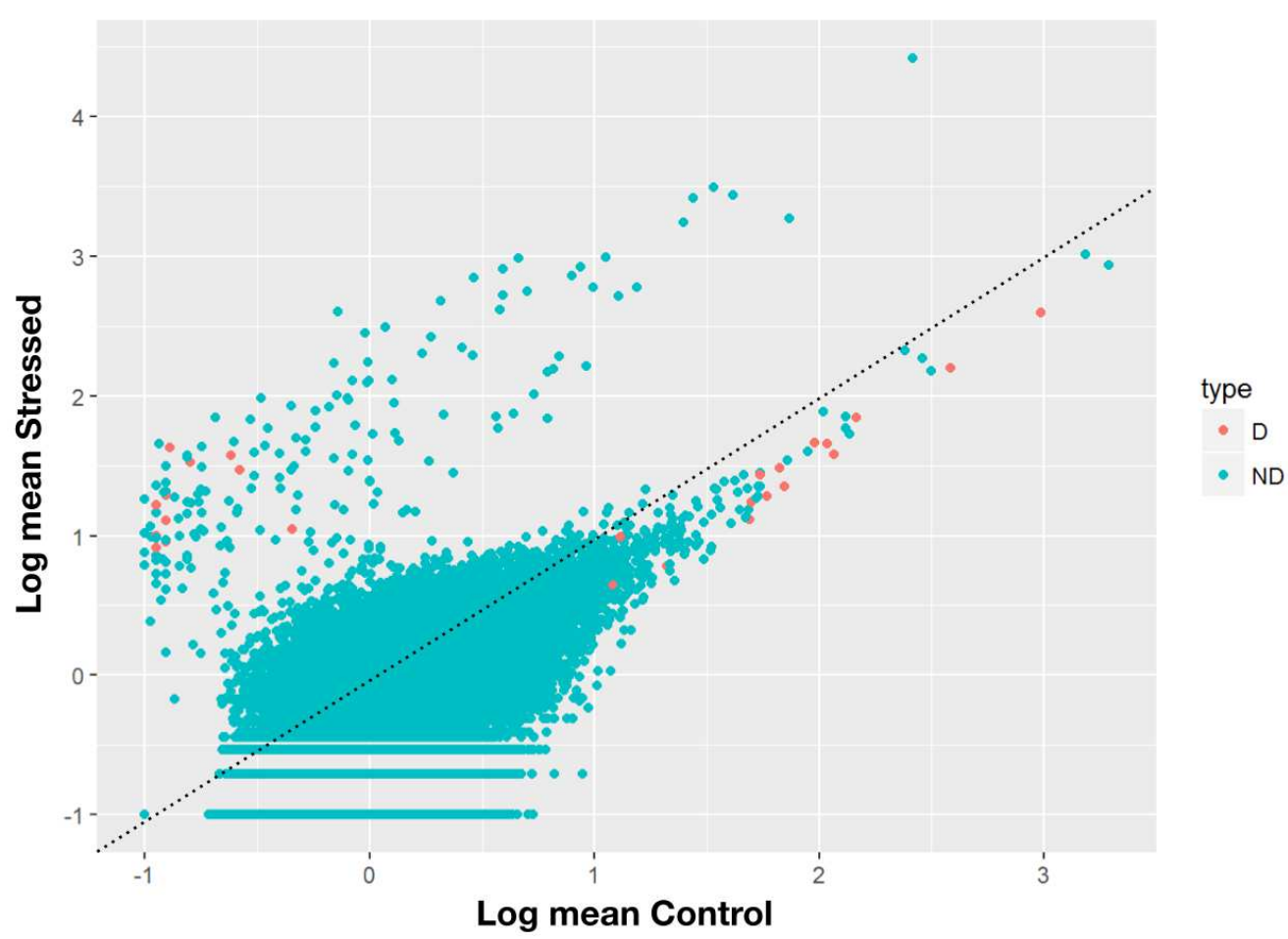

In red = regions differentially accessible according to chromstar.

Figure 7: Plot of transformed ATAC-Seq read counts of 'stressed' vs 'control'. Two point clouds of points: 1 ) at the top-left, those regions have many more reads in stressed than in controls $(+1-2 \log 10$ units $=10$ 100fold change), and 2) under the 1:1 stressed-control dotted line, those regions that are slightly less represented in the 'stressed' than in the 'controls'. Red dots (D) represent regions that were determined as differently accessible by ChromstaR HMM approach. ND all others. 


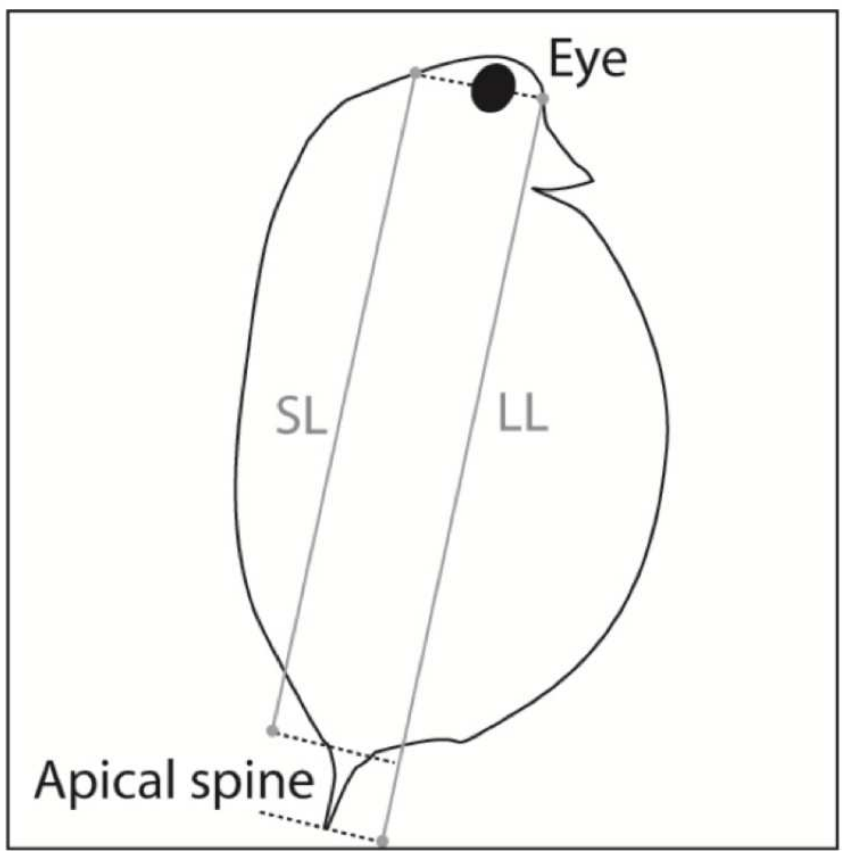

Figure 8: Schematic representation of the measures taken on daphnia. $\mathrm{SL}=$ short length, $\mathrm{LL}=\mathrm{Long}$ length. 
Table 1: Correspondence between sample names used in the text and accession numbers.

\begin{tabular}{|l|l|}
\hline Accession & Sample Name \\
\hline SAMN13189769 & Start S1 \\
\cline { 2 - 2 } SAMN13189770 & Start S11 \\
\hline SAMN13189771 & Start S3 \\
\hline SAMN13189772 & Start S8 \\
\hline SAMN13189773 & Control 11B \\
\hline SAMN13189774 & Control 11C \\
\cline { 2 - 2 } SAMN13189775 & Control 2C \\
\hline SAMN13189776 & Control 4C \\
\hline SAMN13189777 & Control 5A \\
\hline SAMN13189778 & Control 5B \\
\hline SAMN13189779 & Control 8B \\
\cline { 2 - 2 } SAMN13189780 & Control 8C \\
\hline SAMN13189781 & Stress 10B \\
\hline SAMN13189782 & Stress 10C \\
\hline SAMN13189783 & Stress 1C \\
\hline SAMN13189784 & Stress 4A \\
\cline { 2 - 3 } & Stress 4B \\
\hline SAMN13189785 & Stress 5C \\
\hline SAMN13189786 & Stress 7B \\
\hline SAMN13189787 & Stress 7C \\
\hline SAMN13189788 & \\
\hline
\end{tabular}




\section{Figures}

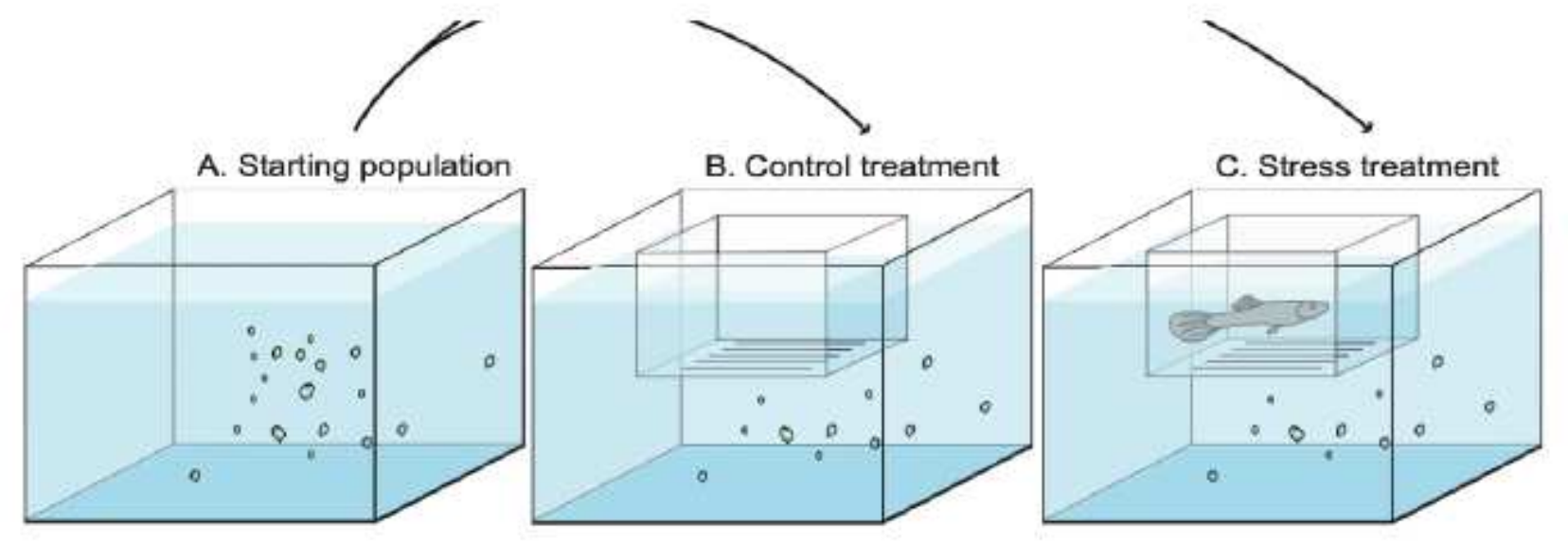

Figure 1

Experimental design used in this study. Daphnia were put into a water tank and allowed to acclimate (start population). Then, two experimental tanks were set up following strictly the same design. The only difference was the presence of a predator (a guppy trained to eat daphnia) in the floating plastic fish breeding isolation box in the stress treatment.

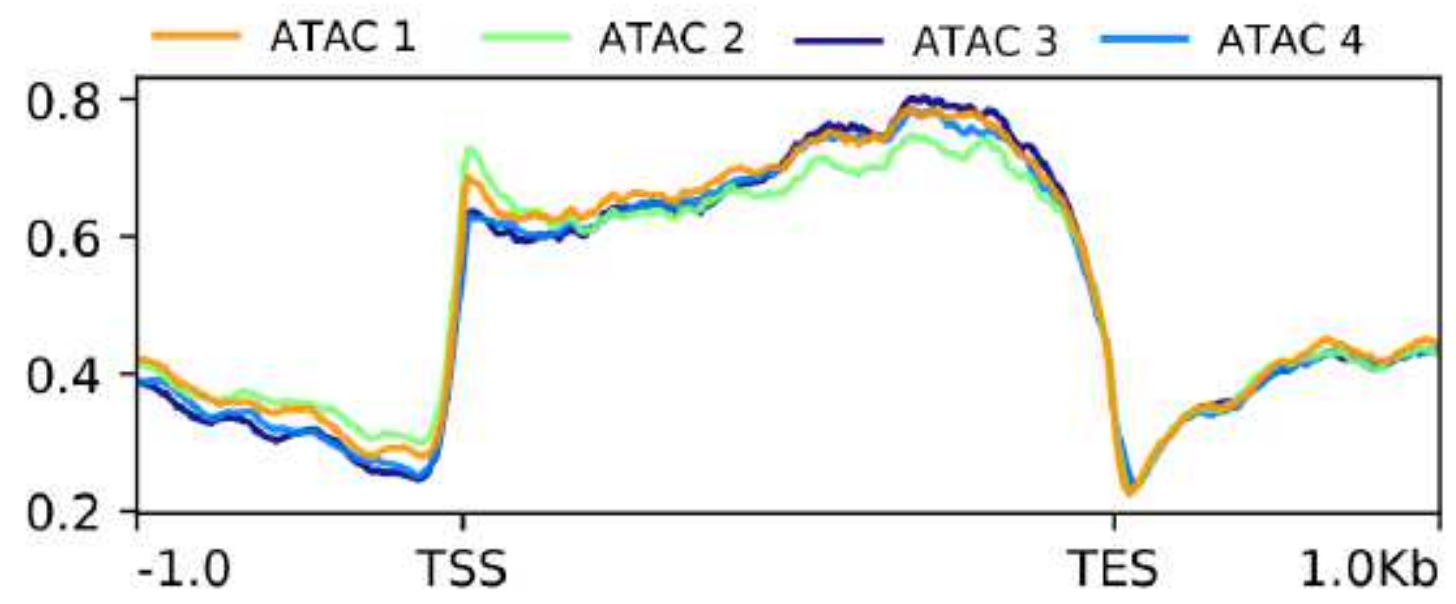

\section{Figure 2}

Superposed metagene ATAC profiles of four individual daphnia of the start populations. X-axis in basepairs. TSS $=$ Transcription start site, TES = transcription end site. Y-axis average enrichment of ATACseq 
reads over genes and upstream and downstream regions. Enrichment of accessible chromatin occurs along the entire length of the genes.

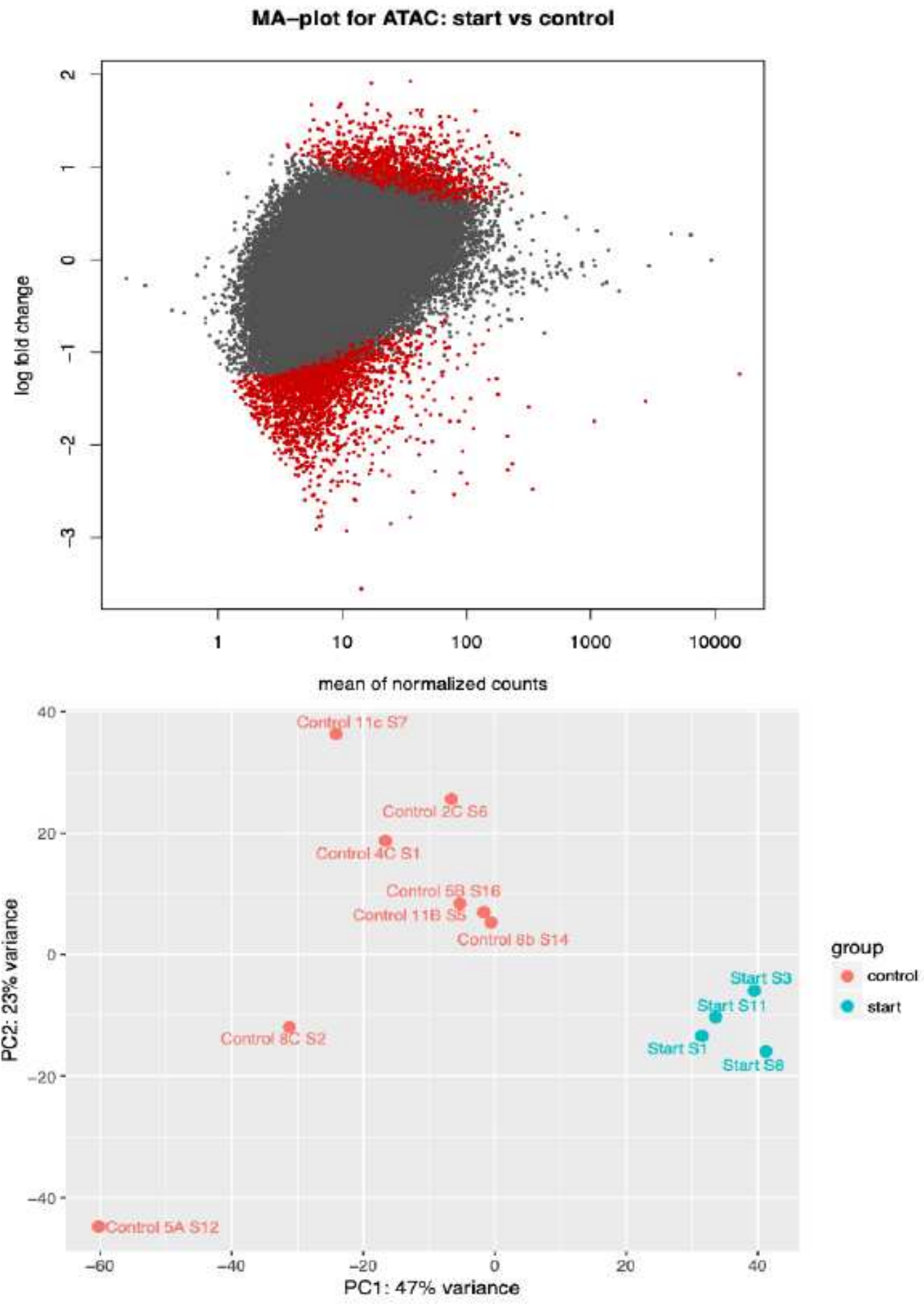

Figure 3

MA-Plot (top) and Principal component analysis of individual Daphnia based on their ATACSeq profiles (bottom). On the PCA every point represents an individual daphnia. Populations are color coded. Samples from the start (blue) and control population (red) cluster clearly. 


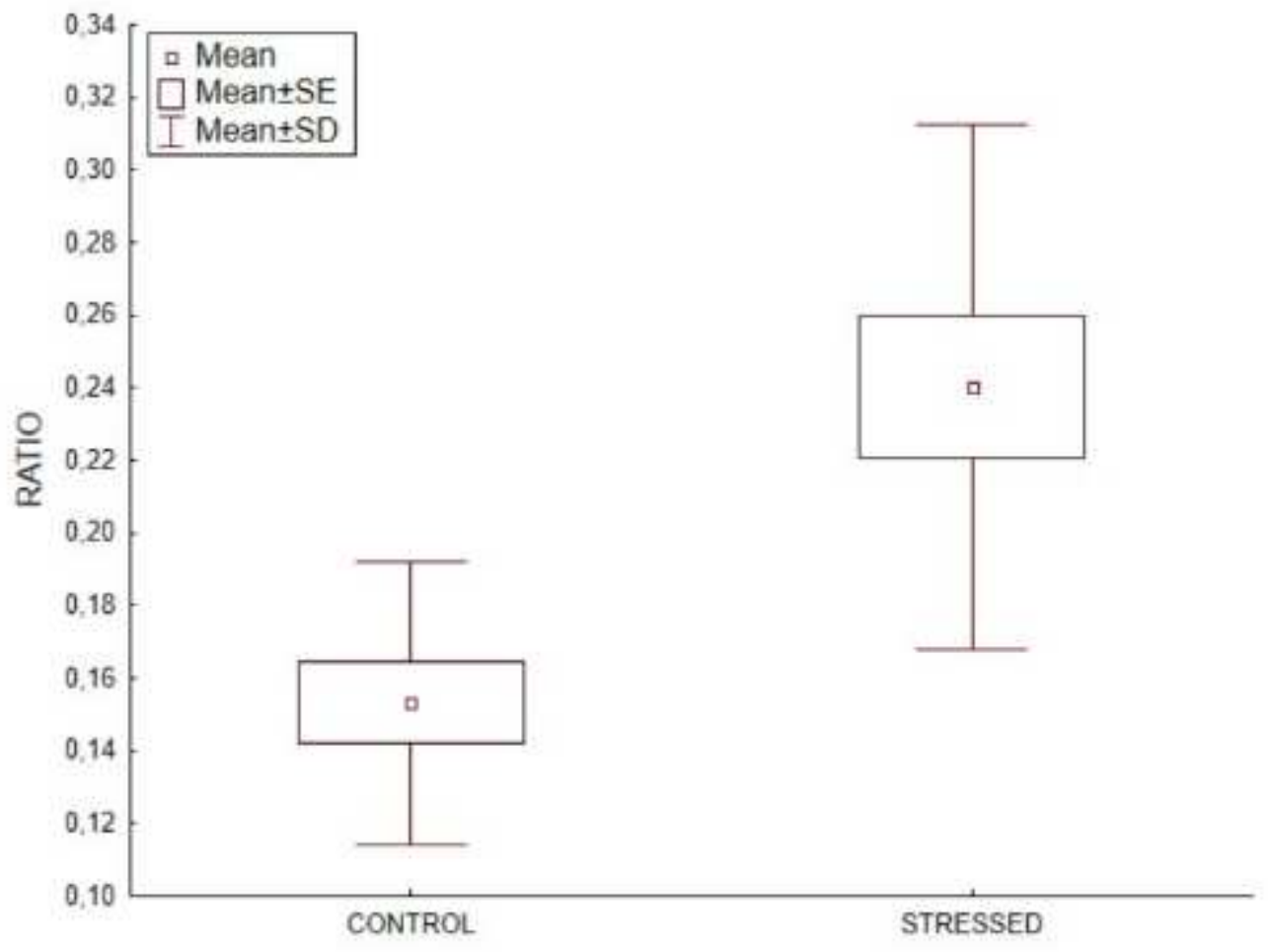

Figure 4

Boxplot of morphometric ratios of (LL-SL)/SL in control and stressed daphnia populations (control: $\mathrm{N}=$ 12; stress: $\mathrm{N}=14$ ). 


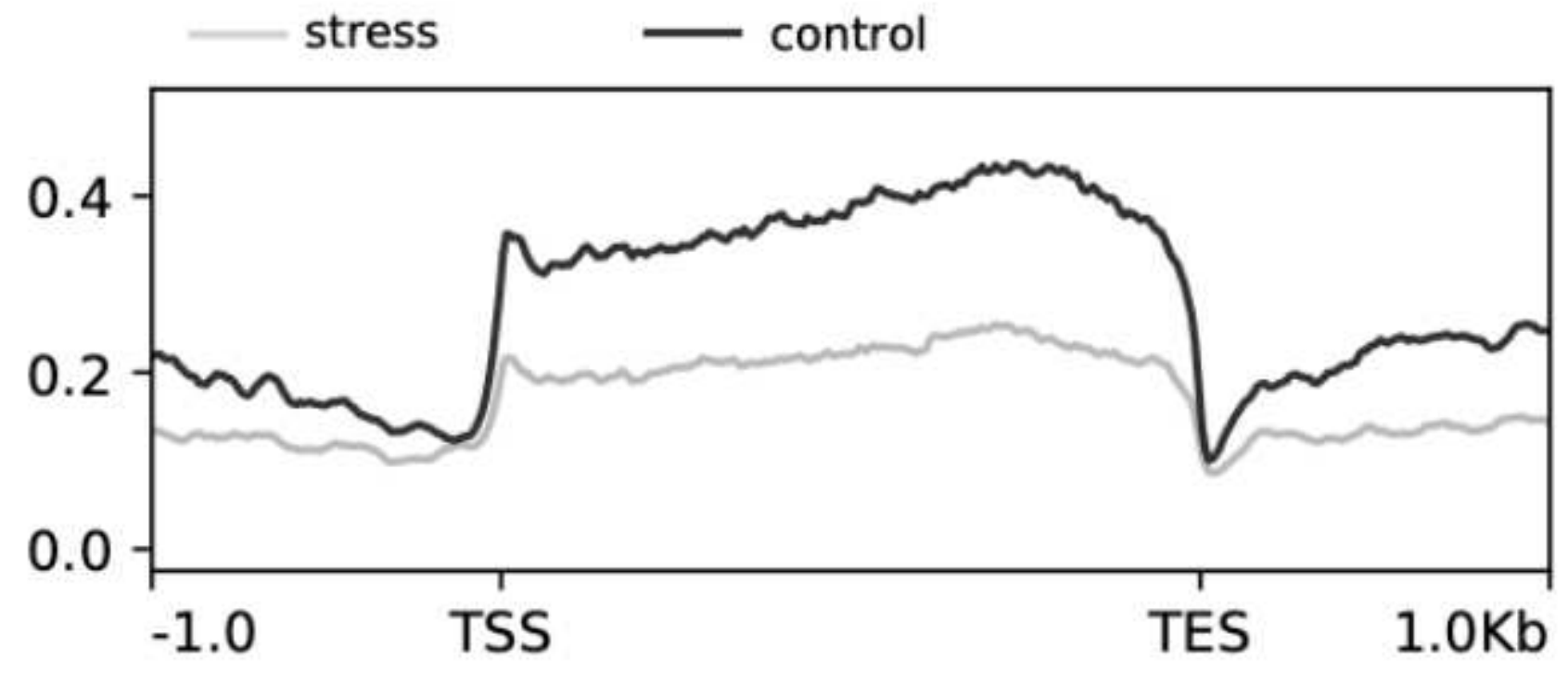

Figure 5

Combined metagene ATAC profiles of stressed and control daphnia populations. X-axis in basepairs. TSS = Transcription start site, TES = transcription end site. Y-axis average enrichment of ATAC-seq reads over genes and upstream and downstream regions. 


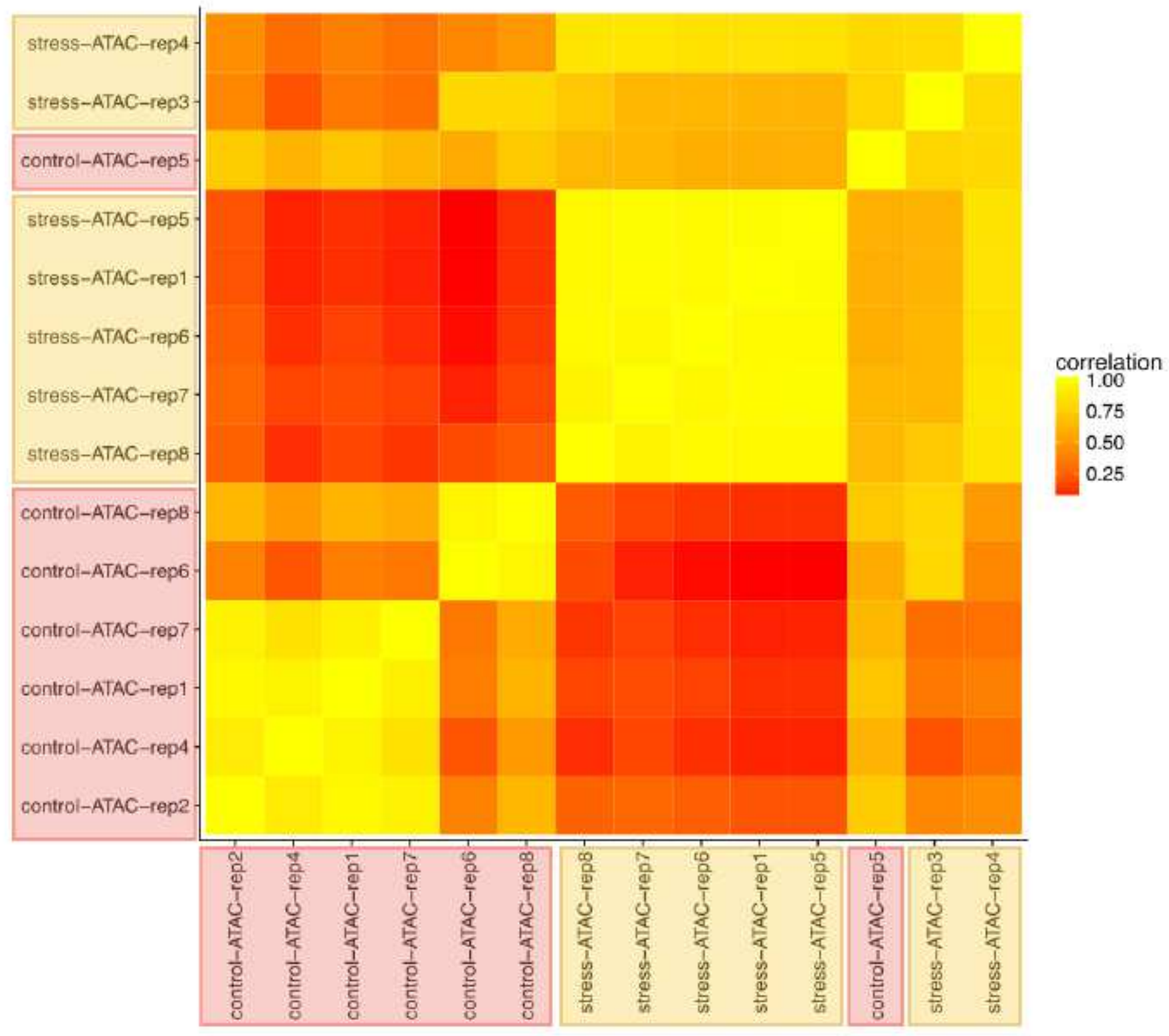

Figure 6

Clustering of individual daphnia based on their ATAC-Seq profiles. Heat map indicating similarity in the HMM ChromstaR results. Generally samples from the stressed and the control populations each cluster together. 


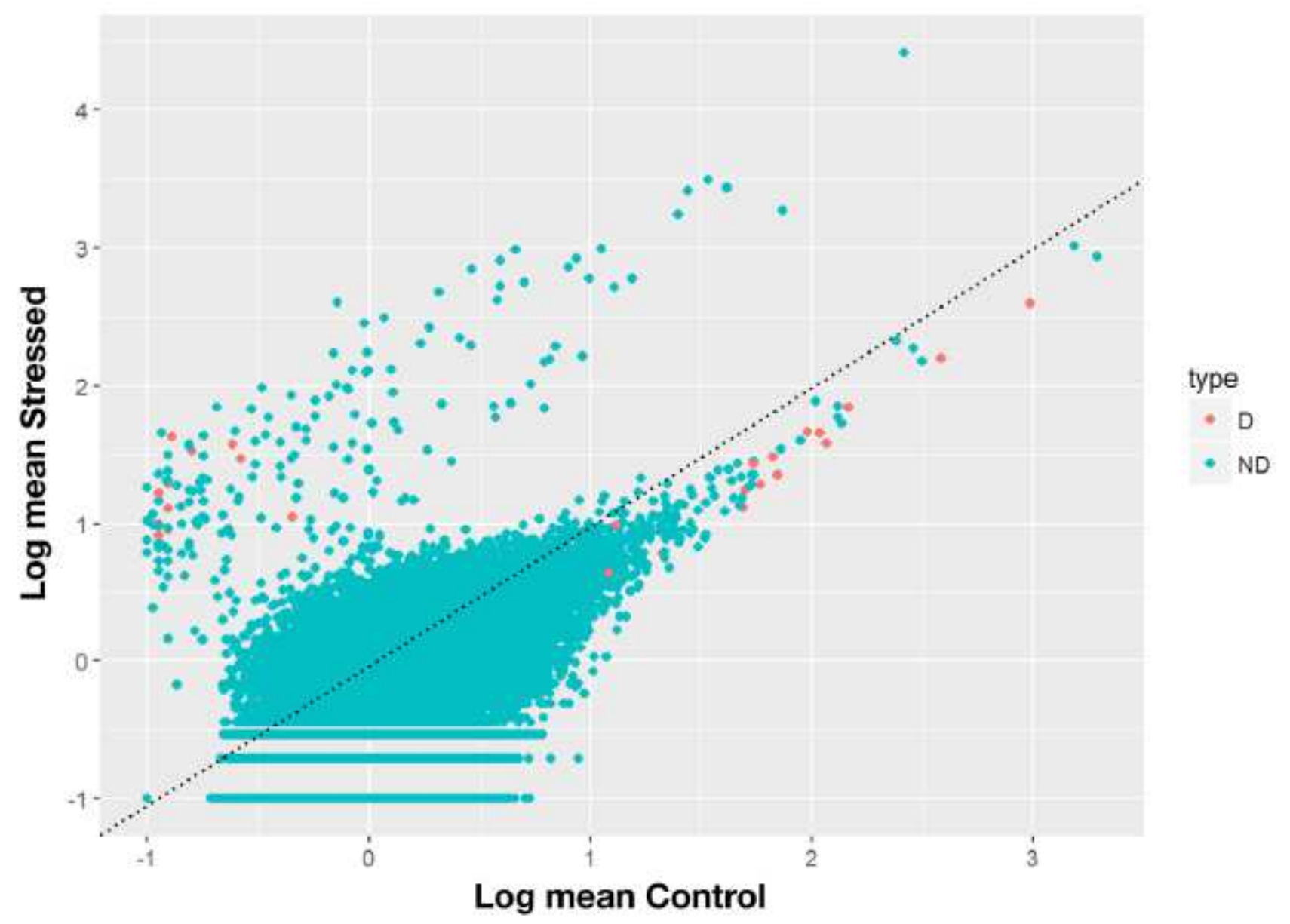

In red = regions differentially accessible according to chromstar.

\section{Figure 7}

Plot of transformed ATAC-Seq read counts of 'stressed' vs 'control'. Two point clouds of points: 1) at the top-left, those regions have many more reads in stressed than in controls $(+1-2 \log 10$ units $=10-100$ fold change), and 2) under the 1:1 stressed-control dotted line, those regions that are slightly less represented in the 'stressed' than in the 'controls'. Red dots (D) represent regions that were determined as differently accessible by ChromstaR HMM approach. ND all others. 


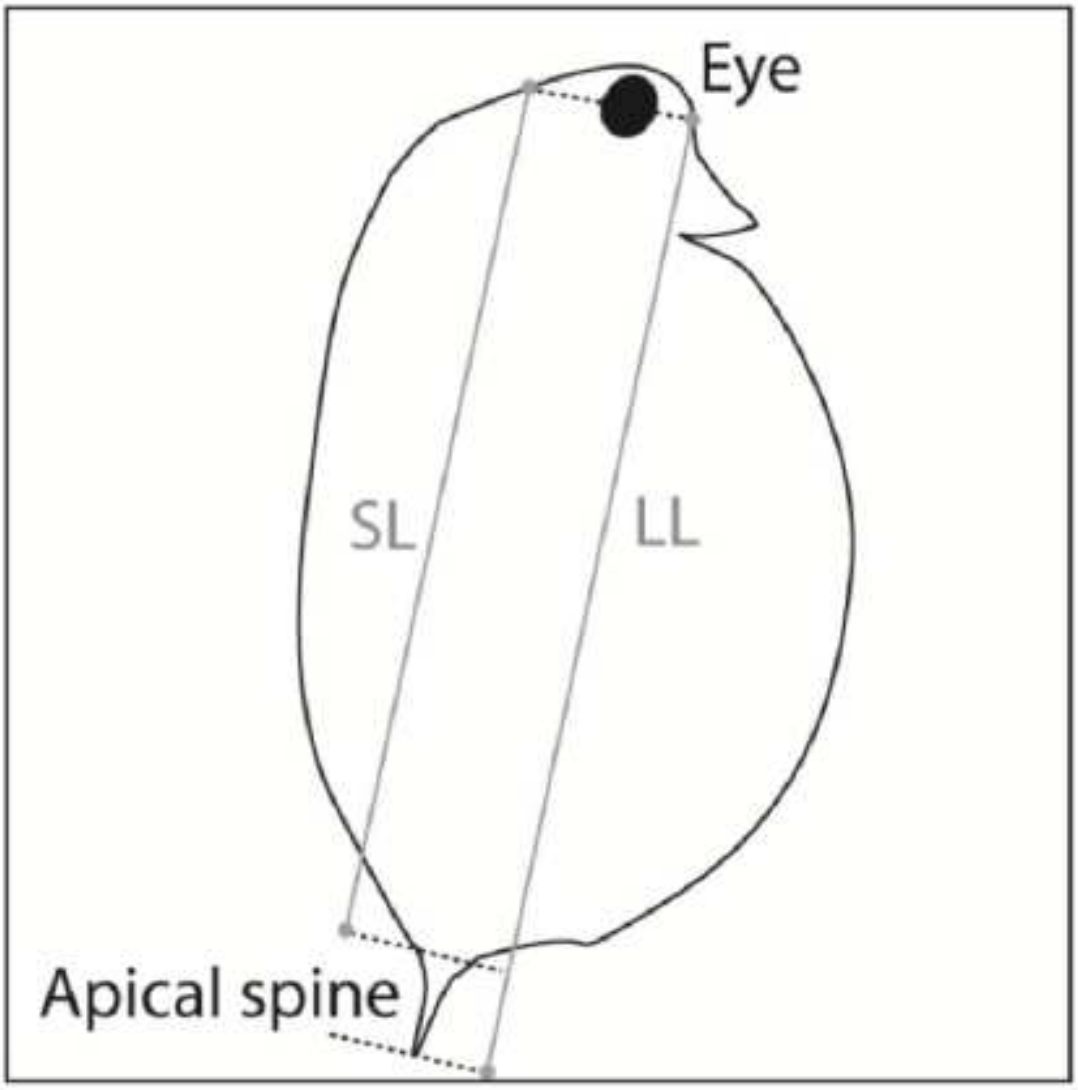

Figure 8

Schematic representation of the measures taken on daphnia. SL = short length, $L L=$ Long length.

\section{Supplementary Files}

This is a list of supplementary files associated with this preprint. Click to download.

- supplementaryfile1profiles.pdf

- supplementaryfile2insertsizestartcontrol.pdf 